American Medical Journal 3 (2): 67-74, 2012

ISSN 1949-0070

(C) 2012 Science Publications

\title{
Intubation using the Video Laryngoscope
}

\author{
${ }^{1}$ Ben H. Boedeker, ${ }^{2}$ W. Bosseau Murray and ${ }^{1}$ Thomas A. Nicholas \\ ${ }^{1}$ Department of Anesthesiology, \\ University of Nebraska Medical Center, Omaha, NE, 68198, USA \\ ${ }^{2}$ Clinical Simulation Center, \\ Penn State College of Medicine, Hershey, PA, 17033, USA
}

\begin{abstract}
Problem statement: Inadequate airway management is a major contributor to morbidity and mortality in the prehospital and out-of-operating room hospital settings. Difficulties that occur during airway management are usually related to the intubator not being able to achieve an adequate view of the glottic opening to allow insertion of an endotracheal tube. Approach: Obtaining an adequate glottic view is technically challenging for the experiened practioner but it is exponentially more difficult for the novice intubator. Needless to say, it is a challenge to teach the actual process of intubation using traditional direct laryngoscopy. Results: Subsequently, numerous intubation devices like the video laryngoscope have been developed that incorporate an optical fiber image transmitter integrated with a modified laryngoscope blade to facilitate visualization of the glottic opening. Conclusion: With the addition of these devices, which have a short, more beneficial learning curve, the video laryngoscope has emerged as a leading method of obtaining an improved view of the glottis opening during intubation.
\end{abstract}

Key words: Video laryngocopy, requiring reintubation, database demonstrates, significant proportion, bullard laryngoscope

\section{INTRODUCTION}

Inadequate airway management is a major contributor to morbidity and mortality in the prehospital and out-of-operating room hospital settings. Difficulties that occur during airway management are usually related to the intubator not being able to achieve an adequate view of the glottic opening to allow insertion of an endotracheal tube. In previous studies, the videolaryngoscope has been shown to improve the view of the glottic opening by at least one Cormack-Lehane grade (Fig. 1) as compared with standard direct laryngoscopy (Cooper et al., 2005; Kaplan et al., 2002; 2006a; 2006b; Cooper, 2003; Rai et al., 2005; Sun et al., 2005; Cormack and Lehane,1984). Video laryngoscopy offers a solution for managing airways especially when performed by personnel whom infrequently intubate patients. It is likely that video laryngocopy will become the standard of care for airway management in the future.

The problem: Inadequate airway management is a major contributor to morbidity and mortality in the prehospital and out-of-operating room hospital settings.
Helm et al. (2006) have reported that several studies examining prehospital deaths from trauma in the United Kingdom have shown that airway obstruction was thought to have contributed to death from major trauma in up to $85 \%$ of patients who died (Helm et al., 2006; Hussain and Redmond,1994). Mort (1998) reported that in patients requiring reintubation outside the operating room after an unplanned tracheal extubation, there was an incidence of difficult laryngoscope in $16 \%$, difficult intubation in $14 \%$ and esophageal intubation in $14 \%$. Less than one third of the patients he studied underwent a mishap-free reintubation.

An analysis by the medical advisory secretariat of Canada found that rates of difficult intubation in the operating room are slightly lower (1.5-3\%) than in the emergency room (3-5\%) or out in the field $(3-10 \%)$. They speculated that the lower rate of difficult intubation in the operating room was because the operating room is a relatively controlled environment, the patient is often unconscious during intubation attempts and the intubation is performed by anesthetists who perform this procedure routinely MAS (2004).

The American Society of Anesthesiologists' Closed Claims Project tracks airway management problems that lead to anesthesia malpractice claims. 


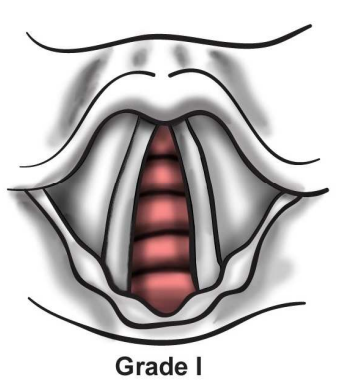

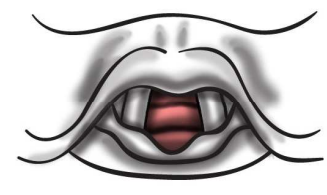

Grade II

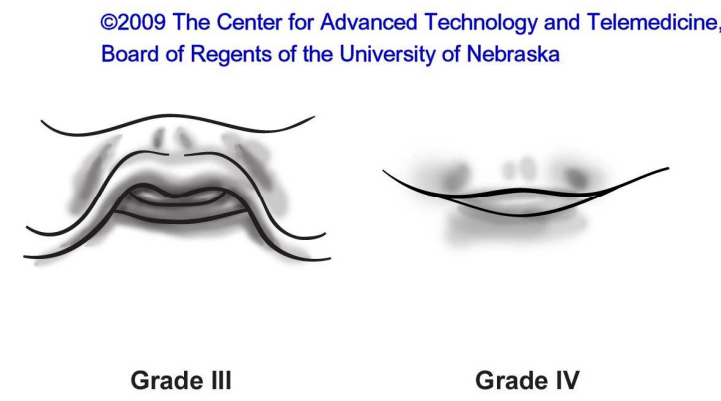

Fig. 1: Cormack-Lehane views of the glottic opening. Grade 1, full view; Grade 2, posterior portion is visible; Grade 3 , only the tip of the epiglottis is visible; Grade 4, only the epiglottis is visible.

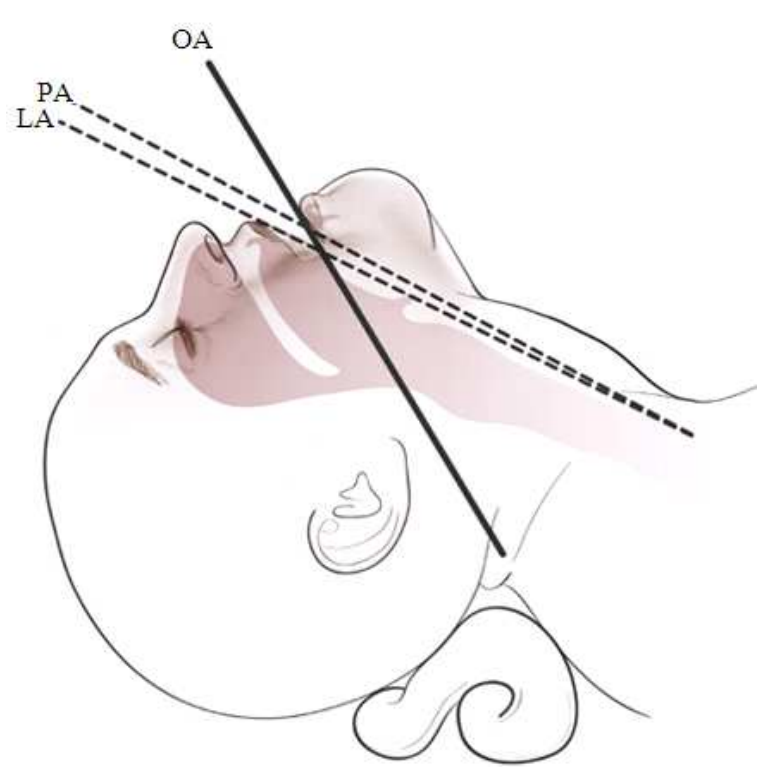

Fig. 2: Alignment of the oral (OA), laryngeal (LA) and pharyngeal (PA) axes must be accomplished during intubation to allow visualization of the glottic opening from the mouth

Miller (2000) this database demonstrates that difficult intubation is the second most frequent primary damaging event leading to anesthesia malpractice claims. It is responsible for $6.4 \%$ of 4,459 claims in the closed claims database. Not only does difficult intubation lead to a significant proportion of claims, the severity of outcome can be devastating. Brain damage or death was the outcome in $57 \%$ of the 283 claims involving difficult intubation Miller (2000).

Why is airway management and intubation so Difficult? Difficulties that occur during airway management are usually related to the intubator not being able to achieve an adequate view of the glottic opening to allow insertion of an endotracheal tube. Review of the anatomy related to intubation shows why obtaining an adequate view of the glottic opening is often difficult. In order to attain a view of the glottic opening from the patient's mouth, the oral, pharyngeal and laryngeal axes must be aligned (Fig. 2). This is anatomically very difficult in many patients, such as those with limited cervical spine mobility, large tongues, short necks of large circumference, protruding incisors, small mandibles, cervical trauma, or other abnormalities of the neck (such as high, anterior or immobile/fixed larynx), or mouth. In the out-ofoperating room cases, the victim's neck is often in a cervical collar and the present advice and management is to avoid extension of the neck. This creates a problem for visualizing the glottic opening of the patient, as the axes cannot be readily aligned.

A possible solution: Numerous intubation devices have been developed that incorporate an optical fiber image transmitter integrated with a modified laryngoscope blade to facilitate visualization of the glottic opening.

These rigid fiber optic laryngoscopes offer the advantage of providing a non-line-of-sight view of the airway and visual control of end tracheal tube advancement through the vocal cords Cooper et al. (2005). Examples include the Bullard laryngoscope (Gyrus ACMI, Inc, Southborough, MA), the WuScope (Achi Corporation, San Jose, CA) and the UpsherScope Ultra (Mercury Medical, Clearwater, FL) (Cooper et al., 2005; Kaplan et al., 2006a; Weiss et al., 2001a; 2001b; Wu and Chou, 1994). These devices have been shown to be effective in providing visualization of difficult airways, but they have a long and difficult learning curve, which results in abandonment by many prospective operators before they acquire competence (Cooper et al., 2005; Jenkins et al., 2002) 


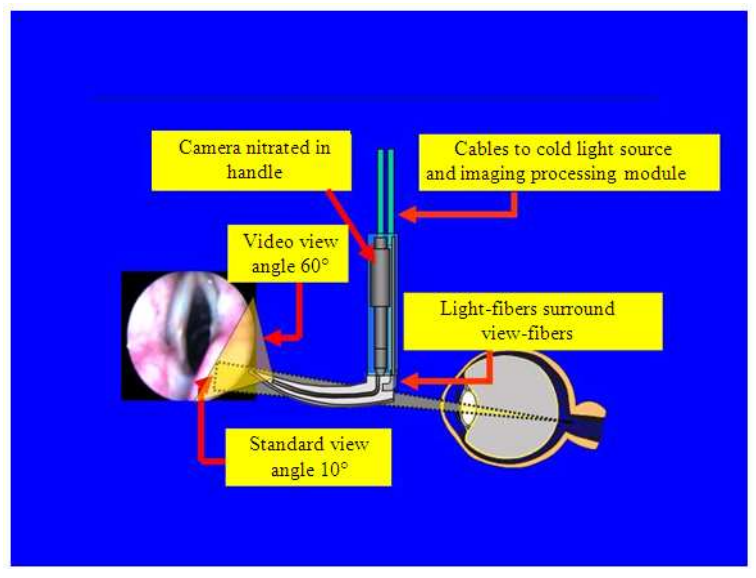

Fig. 3: Visualization of the airway using indirect laryngoscopy

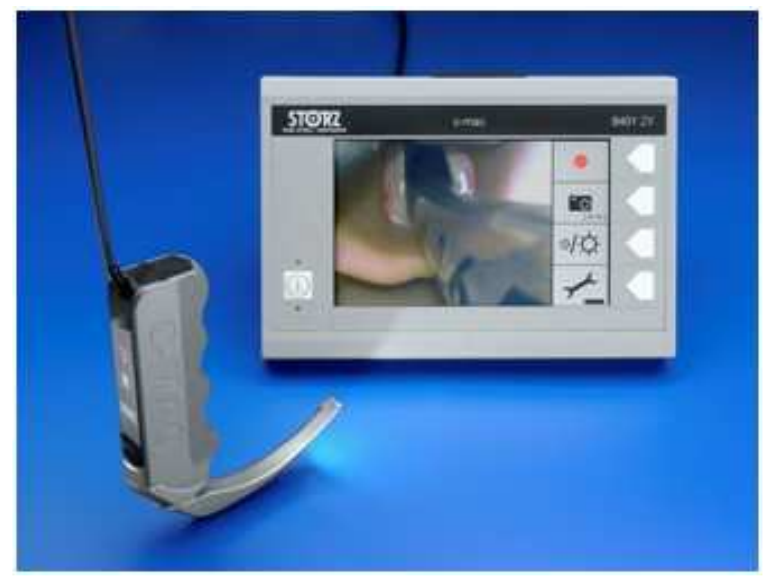

Fig. 4: The Storz CMAC

More recent additions to the field of fiber optic laryngoscope include laryngoscopes that have similar shapes as traditional laryngoscopic blades but with cameras or a lens to transmit images through a fiber optic bundle located on their distal tips. This image is projected through a conduit system (wired or wireless) to a monitor seen by the operator.

With the addition of these devices, which have a short, more beneficial learning curve, the video laryngoscope has emerged as a leading method of obtaining an improved view of the glottis opening during intubation.

The Storz Tele Pack video laryngoscope intubation system (Karl Storz Endoscopy-America, Culver City, CA) was a pioneer advancement for the field of video laryngoscope. This device includes a detachable camera which can be placed inside the laryngoscope handle.

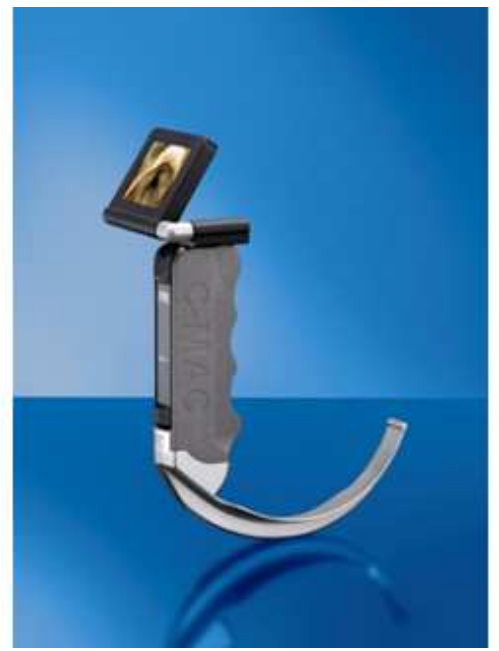

Fig. 5: The Storz C-MAC PM

The camera interfaces with a fiber optic bundle, which runs to the distal tip of the blade, thereby allowing the laryngoscopist to "see around the corner" by using this indirect view. As shown in Fig. 3, the glottis view by classic direct visualization offers a 10-degree view as compared to a much wider 60 -degree view projected by the lens on the video laryngoscope. The camera head is detachable and can be used to interface with several other compatible devices such as rigid stylets, straight or curved laryngoscope blades and fiberoptic bronchoscopes.

Second generation Karl Storz video laryngoscopes have much better resolution, more mega-pixels and less over saturation. These include the STORZ C-MAC (Karl STORZ Endoscopy, Tuttlingen, Germany (Fig. 4) which is more portable than the Telepack and the CMAC PM (Karl STORZ Endoscopy, Tuttlingen, Germany) (Fig. 5) which provides a small viewing screen attached directly to the laryngoscope handle. These devices incorporate a CMOS video camera in the distal end of the video laryngoscope in contrast to fiberoptic technology. The technology change from CCD to C-MOS means the device is also suited to pre hospital airway management, as it is robust against changing environmental conditions such as temperature, dust, moisture and day light.

Advantages of video laryngoscope: Video laryngoscope offers several advantages for airway management.

This technique offers an expanded and enlarged view of the glottic opening. The displayed anatomy is magnified, making recognition of normal anatomy easier, as shown in Fig. 6 Kaplan et al. (2002). 

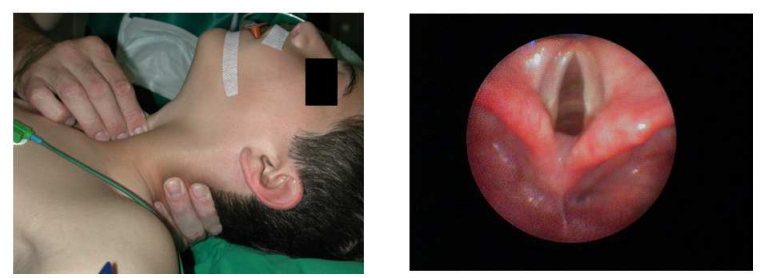

Fig. 6: External Laryngeal Manipulations the assistant performing laryngeal manipulations to improve the view of the glottic opening can determine from the view displayed on the monitor (right) how to best perform this action to achieve the best glottic exposure.(Photograph courtesy of Volker Dorges, MD, University of Kiel, Kiel, Germany)

If laryngeal manipulations must be performed to improve the glottis view, the assistant performing these maneuvers can see on the monitor what impact this effort is having on the view. This greatly coordinates efforts between the laryngoscopes and the assistant performing laryngeal manipulations, as shown in Fig. 6.

When a trainee intubator is attempting to intubate a patient, the video laryngoscope allows the instructor to directly observe the view the trainee has attained and assess his or her actions in manipulating the airway. This enables improved mentoring of the trainee and decreases the chances of the trainees damaging the airway during intubation attempts, which might make the patient unable to be ventilated or intubated Kaplan et al. (2002).

If the airway exceeds the intubation skills of the student, the more experienced laryngoscopist can recognize the difficulty early in the intubation process and intervene.

Projection of the image of the airway to the video monitor facilitates teaching of multiple students who may be observing this procedure.

Pictures and/or videos of the image can be taken with Karl Storz video laryngoscopes for documentation of proper intubation or recording of pathologic findings during intubation (for instance, burns to the vocal cords after an explosion).

The Medi Pack (Karl Storz Endoscopy, Tuttlingen, Germany) can allow video with sound input and output, which enables it to serve as a telemedicine platform if interfaced via a videoconferencing communication advantage of telementoring (in the future). The CMAC also allows video output and can be connected via the $\mathrm{C}$ HUB to a computer as shown in Fig. 7.

The newer video laryngoscopes provide color graphics.

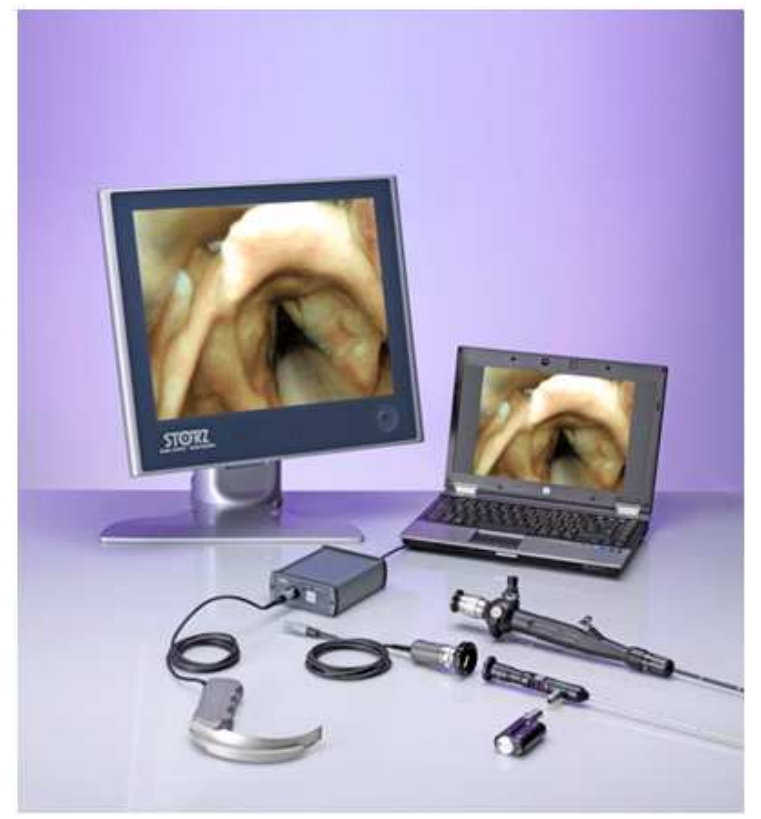

Fig. 7: The Storz C-HUB

The newer video laryngoscopes provide higher resolution (more megapixels) for better viewing.

The relative size of objects: the CMAC shows the tip of the CMAC blade in view at all stages-this provides an immediate reference to the size of objects (which can be distorted due to the camera)

The relationship of tip to anatomical structures: The users always know where the tip of the CMAC blade is, as it is visible in the picture. This helps to know exactly in which direction to move the tip of the blade to obtain a better view. This is in contrast to many other video-devices where the tip of the blade is NOT in view-more difficult to know if the tip of the blade is exactly in the vallecula, or in the mid-line, of determine ("follow") the effect of any movement of the tip of the device.

The newer videolaryngoscopes have less oversaturation (less white-out).

The videolaryngoscope can be used with an intubation stylet (gum elastic bougie). After the intubation stylet, one can watch the endotracheal tube go in, avoiding trauma to the vocal cords.

With the video laryngoscope, there is minimized levering on the upper teeth, as the view is better (direct line of sight is not needed).

With the video laryngoscope, Yankhauer suction is the correct shape for suction using the CMAC blade.

With video laryngoscope, pictures and/or videos are ideal for post-event debriefing and discussion. 


\section{Can demonstrate:}

- too shallow (in terms of vallecula-cannot flip the epiglottis up)

- too deep in terms of vallecula cannot flip epiglottis up (actually pushing it down)

- can see if not midline

- can see if endotracheal tube is advanced incorrectly and goes off the right vocal cord into the paravocal space

With the videolaryngoscope, the intubator does NOT have to be at the head of the bed with the patient at a very specific height (or in the flexion position); the intubator can be in an unusual postion and still have a good chance of intubating successfully. Boedeker et al. (2009) this is useful for patients in entrapped positions, lying on the ground.

Consider the trainee learning classical direct MAC type intubation: While the instructor has the screen turned away from the trainee: As the camera on the videolaryngoscope is near the tip of the blade and also slightly to the left of the view the trainee is seeing, the instructor sees MORE than the trainee (as the trainee is looking into the mouth and the tube typically obscures the right vocal cord just as the trainee is trying to advance the tube through the vocal cords. As the bevel of the typical tube is pointing left (with the sharp tip on the right), there is a tendency for the trainee to "let the tip/bevel slide off" the right vocal cord into the lateral space. Many times, the trainee perceives this as "The tube is too big." And requests a smaller tube. However, the view from the video laryngoscope clearly indicates that the tip of the tube is NOT through the vocal cords, but is impinging on the tissues lateral to the right vocal cord.

With video laryngoscope, the direction of endotracheal tube insertion is a "straight shot". This is due to the shapes of the MAC blade and the endotracheal tube being similar. The tube passes straight through the vocal cords (does not impinge on the anteriror wall of the trachea; does not need a specially shaped stylet, with a special two-handed/two-person technique to slide ("railroad") the tube off the stylet. Also there is less possibility of trauma to the anterior wall of the trachea with rough "pushing off" the stylet. There is no wasted time while railroading the tube off the stylet with the tip barely through the vocal cords.

In NORMAL patients, the CMAC can be used as an assessment of correct MAC use. When the CMAC blade is used correctly, due to the similar shapes of the standard endotracheal tube and the MAC blade, there is a straight shot right through the vocal cords WITHOUT any need for a stylet.

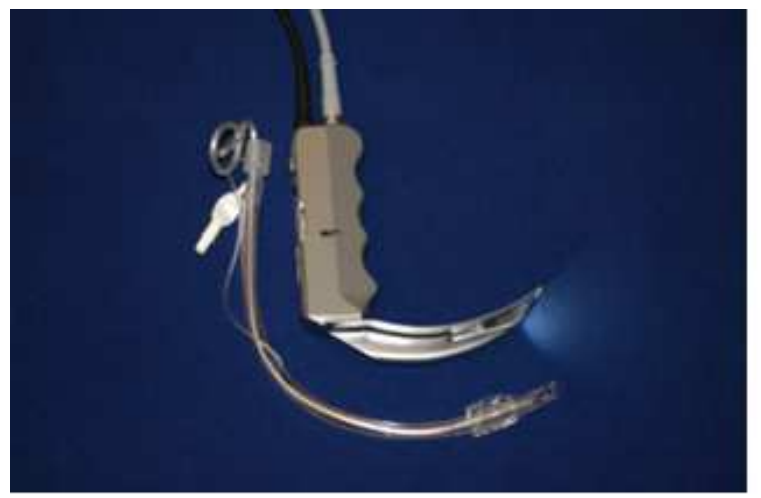

Fig. 8: A stylet can be prebent in the shape of the video laryngoscope blade to facilitate use with an indirect view of a difficult intubation

In difficult intubation patients, a stylet is often needed to enable the tip of the tube to be maneuvered through the vocal cords.

Tips on the use of the video laryngoscope: Insert in Midline over Tongue-the video laryngoscope functions similarly to a "bronchoscope on a stick". Midline insertion (just as you would use a bronchoscope) provides a better view and also provides more room to manuever the endotracheal tube while threading it through the glottic opening.

Also it is easier if one stays in the midline-there is not so much of a tendency to "get lost".

Use Pre Bent Stylet for difficult intubations-The video laryngoscope allows the intubator to "see around the corner", so the view is indirect. The endotracheal tube can be styletted and bent to accommodate placement when working outside of the direct field of vision.

Figure 8 shows pre bending a stylet in the shape of the video laryngoscope blade to facilitate use with an indirect view of a difficult intubation.

Do Not Sweep Tongue-It is not necessary to sweep the tongue when using a video laryngoscope. Sweeping the tongue with a standard laryngoscope is done to create a "keyhole" direct view of the glottis. This smaller working environment makes it more difficult to maneuver the tube through the glottic opening. This is not necessary with a video laryngoscope because the video laryngoscope has a camera on its distal end permitting an expanded, indirect view of the glottic opening. So there is no need to try to achieve a direct line of site.

"Drive" Slowly-Insert the video laryngoscope slowly; keeps structures in view and maintains awareness of airway anatomy as it is advanced. 


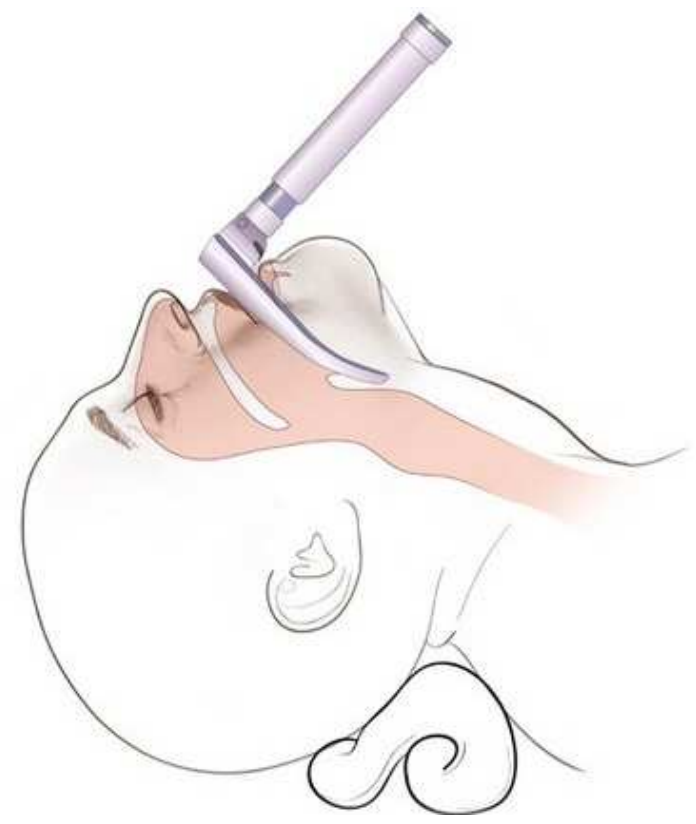

Fig. 9: The curved Macintosh blade

This slow insertion prevents the intubator from losing anatomical landmarks or inserting the blade too deep as is a common problem with inexperienced users of video laryngoscopes. Lift the video laryngoscope slightly anteriorly and drive forward like a bronchoscope recognizing structures as you advance.

For instance, the uvula is easy to find and it clearly indicated exactly where the midline is. By staying in the midline, it is easy to move the short distance from the uvula to the tip of the epiglottis. Advancing anterior to the epiglottis (between the epiglottis and the tongue base) is the most common next step/maneuver (i.e., the MAC mode.) With the tip of the blade in the vallecula, an attempt is made to "flip up" the epiglottis. If the epiglottis is too long and/or floppy, the Miller mode is tried next.

The blade is extracted/withdrawn past the tip of the epiglottis and then advanced posterior to the epiglottis.

The epiglottis is then lifted up in a typical "Miller" mode to expose the vocal cords. Use in Mac or Miller Fashion-A curved (Macintosh) blade (Fig. 9) is commonly inserted in the vallecula when performing direct laryngoscopy. Most video laryngoscopes have a curved blade. However, the videolaryngoscope blade can be used in either the Macintosh fashion (inserting into the vallecula) or in the fashion of a Miller blade (Fig. 10) (by lifting or picking up the epiglottis with the blade tip).

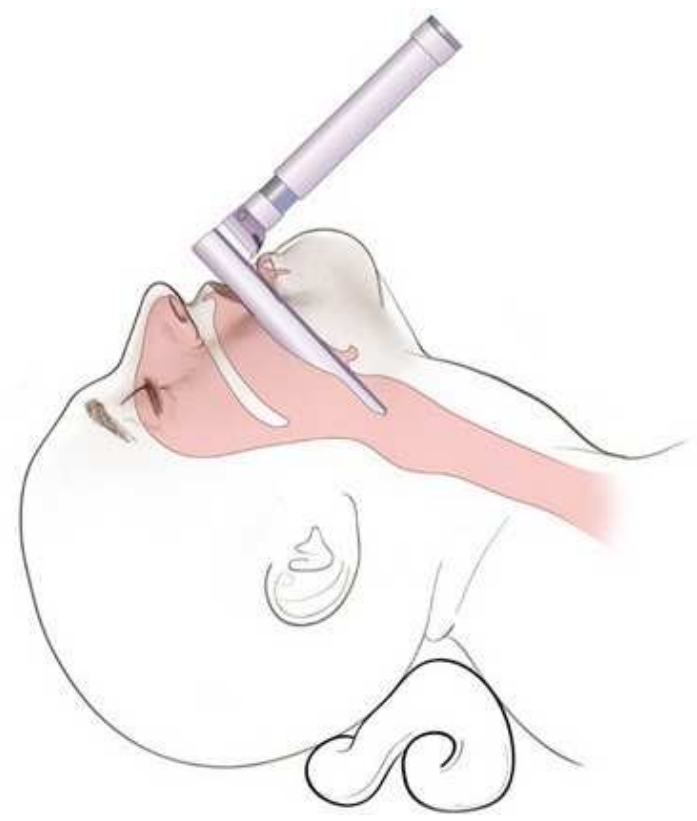

Fig. 10: The straight Miller blade

Visually coordinate laryngeal manipulations: The video laryngoscope offers the advantage of projecting the airway view to a monitor when the operating room team can see the view in addition to the intubator seeing it. This presents an opportunity to train assistants to perform airway manipulation maneuvers (such as cricoid pressure (Fig. 6), adding or removing pillows under the head or shoulders) to improve the airway view. Assistants must be trained to recognize the desired airway view and become more active participants in performing laryngeal manipulations (Fig. 6).

\section{Summary of tips for video laryngoscope:}

- Optimize first attempt

- Suction available-helps improve view from fogging

- Do not sweep tongue

- May use Mac like Miller blade and pick up epiglottis

- Need Stylet-Bend in shape of blade

Use of video laryngoscope for teaching: The video laryngoscope offers advantages for teaching in realtime because both the student and instructor can see the airway view on the monitor screen as shown below. This allows the instructor to point out anatomical landmarks to the students, mentor them as they insert the laryngoscope and perform the intubation. Safety is 
greater because the instructor can constantly see what the student is seeing and observe all portions of the intubation process. If the student is making a mistake, it can be immediately corrected by the mentor. Projection of the airway view onto a monitor also enables more than one student to participate in learing the anatomy and intubation process. This is useful nowadays when there is competition among students for intubation training.

The video laryngoscope offers advantages for teaching off-line instruction/debriefing using pictures and/or videos (Fig. 11).

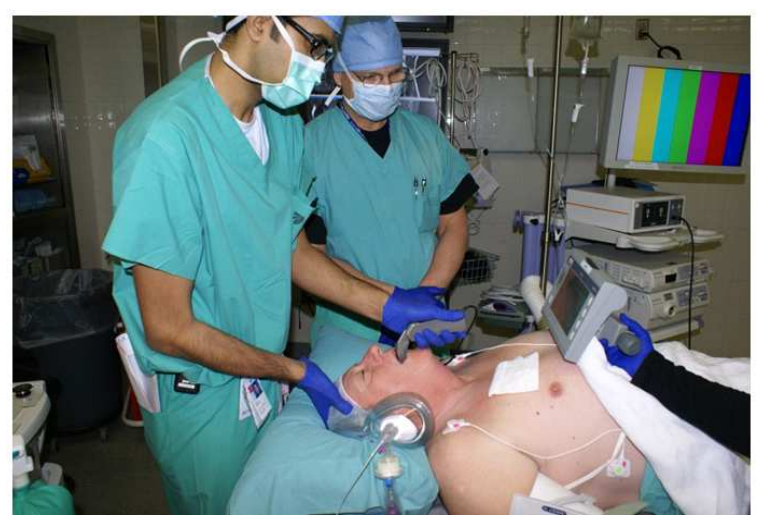

Fig. 11: Intubation training with the videolaryngoscope

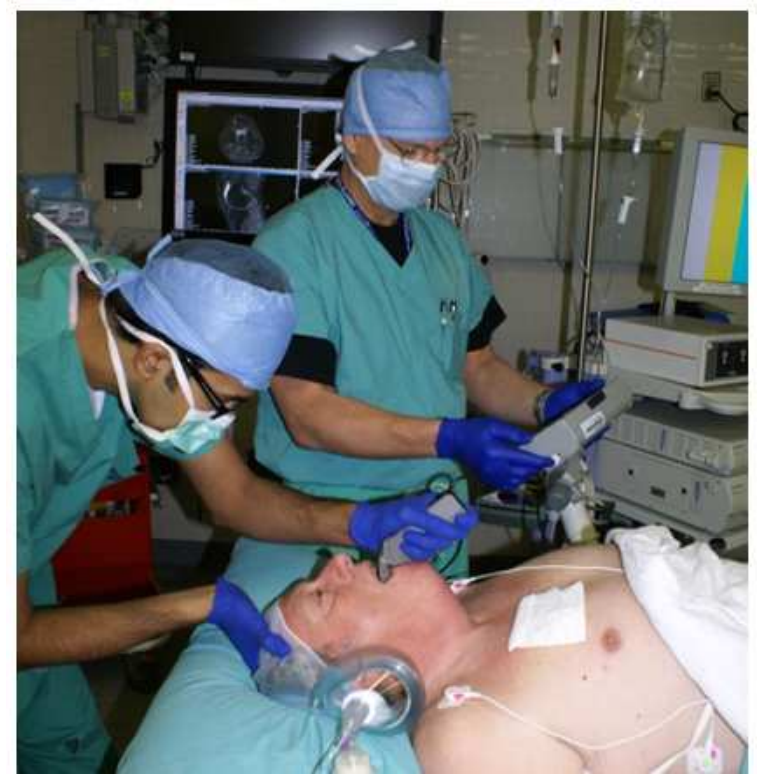

Fig. 12: The screen of the videolaryngoscope can be turned away from the trainee so that others can see the progress of the intubation
Teaching direct intubation with a video laryngoscope: A standard curved (Macintosh) or straight blade can be used with the Karl Storz video laryngoscope series. This allows a student to use a standard shaped curved or straight blade. If the viewing monitor is turned away from the student's view, it creates a training situation where the student is performing a standard, direct laryngoscopy, while the instructor has a full view of the intubation process. This allows the student to learn and practice direct laryngoscopy with greater safety as the instructor is monitoring the entire process (Fig. 12).

\section{CONCLUSION}

In conclusion, it is clear that video laryngoscopy has several advantages: Namely, it is easy to learn and a better teaching modality. The Karl Storz video laryngoscope uses a standard shaped laryngoscope blade which facilitates learning to perform video laryngoscope.

This better teaching modality is mainly based upon the fact that the instructor sees what the student is viewing. Therefore, both the student and instructor have a view of the airway. This facilitates teaching while also improving safety. Immediate modifications in technique can be performed to optimise safety of the patient.

The video laryngoscope provides an improved view of the glottic opening. Numerous studies have shown that there was an improvement of at least one Cormack-Lehane grade (Figure 1) using video laryngoscopy as compared with standard direct laryngoscopy (Cooper et al., 2005; Kaplan et al., 2002; 2006a; 2006b; Cooper, 2003; Rai et al., 2005; Sun et al., 2005; Cormack and Lehane,1984).

Lastly, assistants can provide coordinated laryngeal manipulations by also visualizing the direct effects of their laryngeal manipulations or patient positioning during intubation. Therefore, this sharing of the laryngoscopic view can guide airway manipulations to optimize intubation conditions.

It is evident that video laryngoscopy likely will become the standard of care due to ease of use, enhanced safety and improved teaching modality.

\section{REFERENCES}

Boedeker, B.H., B.W. Berg, M. Bernhagen and W.B. Murray, 2009. Endotracheal intubation in a medical transport helicopter - comparing direct laryngoscopy with the prototype Storz CMAC videolaryngoscope in a simulated difficult intubating position. Stud. Health Technol. Inform., 142: 40-42. PMID: 19377110 
Cooper, R.M., 2003. Use of a new videolaryngoscope (GlideScope $\AA$ ) in the management of a difficult airway. Can. J. Anaesth., 50: 611-613.

Cooper, R.M., J.A. Pacey, M.J. Bishop and S,A. McCluskey, 2005. Early clinical experience with a new videolaryngoscope (GlideScope $\AA$ ) in 728 patients. Can. J. Anaesth., 52: 191-198. DOI: 10.1007/BF03027728

Cormack, R.S. and J. Lehane, 1984. Difficult tracheal intubation in obstetrics. Anesthesia, 39: 1105-1111. DOI: 10.1111/j.1365-2044.1984.tb08932.x

Helm, M., B. Hossfeld, S. Schäfer, J. Hoitz and L. Lampl, 2006. Factors influencing emergency intubation in the pre-hospital setting-a multicentre study in the German Helicopter Emergency Medical Service. Br. J. Anaesth., 96: 67-71. DOI: 10.1093/bja/aei275

Hussain, L.M. and A.D. Redmond, 1994. Are prehospital deaths from accidental injury preventable? Br. Med. J., 308: 1077-1080. DOI: 10.1136/bmj.308.6936.1077

Jenkins, K., D.T. Wong and R. Correa, 2002. Management choices for the difficult airway by anesthesiologists in Canada. Can. J. Anaesth., 49: 850-856. DOI: $10.1007 / \mathrm{BF} 03017419$

Kaplan, M.B., D.S. Ward and G. Berci, 2002. A new video laryngoscope - an aid to intubation and teaching. J. Clin. Anesth., 14: 620-626. DOI: 10.1016/S0952-8180(02)00457-9

Kaplan, M.B., D. Ward, C.A. Hagberg, G. Berci and M. Haglike, 2006a. Seeing is believing: the importance of video laryngoscopy in teaching and in managing the difficult airway. Surg. Endosc., 20: S479-S483. DOI: 10.1007/s00464-006-0038-z

Kaplan, M.B., C. Hagberg, D.S. Ward, A. Brambrink and A.K. Chhibber et al., 2006b. Comparison of direct and video-assisted views of the larynx during routine intubation. J. Clin. Anesth., 18: 357-362. DOI: $10.1016 /$ j.jclinane.2006.01.002
MAS, 2004. Video laryngoscopy for tracheal intubation. Ontario Health Technol. Assessment Series, 4: 1-23.

Miller, C.G., 2000. Management of the difficult Intubation in closed malpractice claims. ASA Newsletter 64: 13-16.

Mort, T.C., 1998. Unplanned tracheal extubation outside the operating room: a quality improvement audit of hemodynamic and tracheal airway complications associated with emergency tracheal reintubation. Anesth Analg., 86: 1171-1176. DOI: 10.1213/00000539-199806000-00006

Rai, M.R., A. Dering and C. Verghese, 2005. The Glidescope ${ }^{\circledR}$ system: A clinical assessment of performance. Anaesthesia, 60: 60-64. DOI: 10.1111/j.1365-2044.2004.04013.x

Sun, D.A., C.B. Warriner, D.G. Parsons, R. Klein and H.S. Umedaly et al., 2005. The GlideScope® Video Laryngoscope: randomized clinical trial in 200 patients. Br. J. Anaesth., 94: 381-384. DOI: 10.1093/bja/aei041

Weiss, M., K. Hartmann, J. Fischer and A.C. Gerber, 2001a. Video-intuboscopic assistance is a useful aid to tracheal intubation in pediatric patients. Can. J. Anaesth., 48: 691-696. DOI: 10.1007/BF03016206

Weiss, M., U. Schwartz, C.M. Dillier and A.C. Gerber, 2001b. Teaching and supervising tracheal intubation in paediatric patients using videolaryngoscopy. Paediatr Anaesth., 11: 343348. DOI: 10.1046/j.1460-9592.2001.00681.x

Wu, T.L. and H.C. Chou, 1994. A new laryngoscope: the combination intubating device. Anesthesiology, 81: 1085-1087. DOI: 10.1097/00000542199410000-00044 